Published in final edited form as:

Support Care Cancer. 2017 August ; 25(8): 2593-2601. doi:10.1007/s00520-017-3670-3.

\title{
Fluoroquinolone prophylaxis in autologous hematopoietic stem cell transplant recipients
}

\author{
Dipenkumar Modi ${ }^{1}$, Hyejeong Jang ${ }^{2}$, Seongho $\mathrm{Kim}^{2}$, Malini Surapaneni ${ }^{3}$, Kamya Sankar ${ }^{4}$, \\ Abhinav Deol ${ }^{5}$, Lois Ayash ${ }^{5}$, Divaya Bhutani ${ }^{5}$, Lawrence G. Lum ${ }^{6}$, Voravit \\ Ratanatharathorn ${ }^{5}$, Richard Manasa ${ }^{7}$, Kendra Mellert ${ }^{7}$, Pranatharthi Chandrasekar ${ }^{8}$, \\ Joseph P. Uberti ${ }^{5}$
}

${ }^{1}$ Department of Hematology-Oncology, Karmanos Cancer Institute/Wayne State University, 4100 John R, HW04H0, Detroit, MI 48201, USA

${ }^{2}$ Biostatistics Core, Karmanos Cancer Institute, Department of Oncology, Wayne State University, Detroit, MI 48201, USA

${ }^{3}$ Department of Internal Medicine, 3990 John R, 5904 5Hudson, Detroit, MI 48201, USA

${ }^{4}$ Wayne State School of Medicine, 320 E Canfield Ave; Suite 315, Detroit, MI 48201, USA

${ }^{5}$ Department of Oncology, Blood and Marrow Stem Cell Transplant Program, Karmanos Cancer Institute/Wayne State University, 4100 John R, 4 HW04H0, Detroit, MI 48201, USA

${ }^{6}$ Division of Hematology/Oncology, University of Virginia Cancer Center, West Complex, Rm 7191, 1300 Jefferson Park Avenue, Charlottesville, VA, USA

${ }^{7}$ Clinical Trials Office Bone Marrow Transplant, Karmanos Cancer Institute, 4100 John R, WN10SC, Detroit, MI 48201, USA

${ }^{8}$ Division of Infectious Diseases, Wayne State University, Detroit, MI 48201, USA

\begin{abstract}
Purpose-Although fluoroquinolone prophylaxis is frequently utilized in autologous hematopoietic stem cell transplant (AHSCT) patients, its impact on morbidity and mortality is uncertain. This study investigates the role of quinolone prophylaxis after AHSCT in recent years.

Methods-We conducted a retrospective review of 291 consecutive adult patients who underwent AHSCT for malignant disorders, between June 2013 and January 2015. Outcomes were compared between patients who received norfloxacin prophylaxis and those who did not. The endpoints were mortality during prophylaxis and at 100 days after transplant, frequency of ICU admissions, and incidence and type of bacteremia.
\end{abstract}

Dipenkumar Modi, modid@karmanos.org; drdipen228@gmail.com.

Conflict of interest The authors declare that they have no conflict of interest.

Declaration We presented our study data at American Society of Blood and Marrow Transplantation (ASBMT) annual meeting 2016. Only abstract of our study was published in Biology of Blood and Marrow Transplantation journal supplement issue in March 2016, and only abstract is available online (Google scholar). 
Results-Of 291 patients, 252 patients received norfloxacin prophylaxis and 39 patients did not. The mortality during prophylaxis and at 100 days as well as the median number of days of hospitalization following AHSCT did not differ between the two groups. No differences were noted in the frequency of ICU admission, incidence of septic shock, and duration of ICU stay. Patients who did not receive prophylaxis had a significantly higher rate of neutropenic fever (97\%) than patients who received prophylaxis $(77 \%)(p=0.005)$. The patients with prophylaxis demonstrated a significantly higher rate of gram-positive bacteremia as compared to those without prophylaxis $(p=0.002)$. Frequency of Clostridium difficile infection was similar during and postprophylaxis. More antibiotic use was noted among patients without prophylaxis [97\%; median 9 (range, 5-24) days] compared to patients with prophylaxis [79\%; median 7 (range, 3-36) days, $p=$ $0.04]$.

Conclusion-Although fluoroquinolone prophylaxis reduced the incidence of neutropenic fever and antibiotic use in AHSCT, it did not alter mortality or morbidity.

\section{Keywords}

Fluoroquinolone prophylaxis; Autologous stem cell transplant; Infection prophylaxis; Neutropenic fever; Length of stay and ICU admission

\section{Introduction}

Neutropenic fever following autologous hematopoietic stem cell transplant (AHSCT) is a major clinical concern with the incidence ranging between 10 and 100\% [1,2], and bacterial infections account for considerable morbidity and mortality in the pre-engraftment setting [3-5]. Neutropenic fever leads to increased antibiotic use and prolonged hospital stay and significantly increases the healthcare cost. Over the years, various prophylactic measures have been utilized to reduce the risk of infection during severe neutropenia, and the most common measure is selective intestinal decontamination with the use of fluoroquinolones [6-8]. Fluoroquinolones are favored owing to their activity against aerobic gram-negative bacilli, inactivity against anaerobic flora in alimentary tract, systemic bactericidal activity, good tolerability, and no bone marrow toxicity $[6,7]$.

Many studies evaluating the efficacy of fluoroquinolone prophylaxis in chemotherapyinduced neutropenia in patients with leukemia, lymphoma, or solid tumors have demonstrated reduction in neutropenic fever, microbiologically documented infections, bacteremia, and hospitalizations $[9,10]$. Few studies have reported association of fluoroquinolone use with the emergence of resistant gram-negative organisms [11-13], Clostridium difficile infection [14, 15], low response to empiric first-line antibiotics [16], and the need for use of extended spectrum antibiotics such as carbapenems [12]. Importantly, the available trials including meta-analyses involving over 14,000 patients demonstrated conflicting results in terms of mortality $[5,17]$. The studies evaluating efficacy and risks of fluoroquinolone prophylaxis in the setting AHSCT are few. Most studies demonstrated reduction in neutropenic fever, bacteremia, and antibiotic use $[11,16,18,19]$; however, detailed information on mortality and morbidity is lacking. Therefore, we performed a retrospective study of AHSCT patients at our institution to evaluate the effects 
of norfloxacin prophylaxis on the morbidity and mortality during prophylaxis and at 100 days.

\section{Materials and methods}

We conducted retrospective study of 291 consecutive adult patients who underwent peripheral blood AHSCT for hematologic malignancies between June 2013 and January 2015 at our institution. This study was approved by the Wayne State University Institutional Review Board (IRB).

We collected demographic and clinical data such as patient characteristics, diagnosis, prior treatment regimens and $C$. difficile infection, disease status at transplant, conditioning regimen, development of neutropenic fever, presence or absence of clinically or microbiologically documented infections, use of antibiotics, need for ICU care, duration of hospital stay, readmission rate, mortality during prophylaxis (at engraftment) as well as at 100 days for the patients who received fluoroquinolone as antimicrobial prophylaxis compared to the patients who did not. The AHSCT recipients are at higher risk of having infections during pre-engraftment period and first 100 post-transplant days. Hence, we especially focused evaluating morbidity and mortality during prophylaxis and at 100 days post-transplant. Norfloxacin is poorly absorbable through GI tract, which provides higher concentration in bowel. Therefore, norfloxacin (400 mg twice a day started on day -1) was the fluoroquinolone used in patients who received antibacterial prophylaxis. Moreover, the decision to administer norfloxacin prophylaxis was based on treating physician discretion. All patients received fluconazole (100 mg daily started on day -1$)$ and acyclovir (400 mg twice daily started on day +1 ) prophylaxis. The antimicrobial prophylaxis was continued until an absolute neutrophil count (ANC) greater than $1000 / \mathrm{mm}^{3}$ was attained or there was development of a febrile episode in which case management was changed to empiric therapy with IV broad spectrum antibiotics. Granulocyte-colony stimulating factor (G-CSF) was started on day +6 and continued till engraftment. Disease status at transplant was divided into chemo-sensitive and chemo-resistant groups. Chemo-sensitive group consisted of patients who were in complete remission (CR), partial remission (PR), very good partial remission (VGPR), and stringent complete remission (SCR). Chemo-resistant group consisted of patients with stable disease (SD), progressive disease (PD), relapse (REL), and primary induction failure (PIF).

Neutropenic fever was defined as oral temperature of $38.0^{\circ} \mathrm{C}$ on two or more occasions over a 12-h period or temperature of $38.5^{\circ} \mathrm{C}$ on a single occasion during the neutropenic period [20]. Neutropenia was defined as ANC $<500 / \mathrm{mm}^{3}$ or ANC $<1000 / \mathrm{mm}^{3}$ and a predicted decline to $500 / \mathrm{mm}^{3}$ or less over the next $48 \mathrm{~h}$ [20]. Engraftment was defined by three consecutive daily ANC of $>500 / \mathrm{mm}^{3}$.

In the event of fever, the data on complete blood count with differential, basic metabolic panel, chest X-ray, urine analysis, as well as results of blood and urine cultures (stool culture, sputum culture, and bronchoalveolar lavage (BAL) results if applicable) were obtained. Per institutional standards, two blood cultures were obtained from the central vascular catheter and a peripheral vein in all patients with neutropenic fever. Blood stream 
infection (BSI) was defined as one or more positive blood culture for any organism. Blood cultures were repeated till two consecutive blood cultures remained negative for $48 \mathrm{~h}$. Etiology of neutropenic fever was broadly divided into clinically documented infection, microbiologically documented infection, and fever of unknown origin (FUO). Clinically documented infection was defined as fever associated with a local inflammation such as pneumonia, skin infection, or cellulitis, whose microbiological pathogenesis cannot be proven or which cannot be examined [21]. Microbiological documented infection was defined as an infectious organism detected in blood cultures, even without localized inflammation (no clinical focus) or localized, microbiologically documented, infection, with or without positive blood cultures [21]. FUO was defined as a temperature $>38.3{ }^{\circ} \mathrm{C}$ $\left(101^{\circ} \mathrm{F}\right)$ on several occasions without identifiable source of infection. These cases could also be considered as culture-negative neutropenic fever. Empiric vancomycin was added in case of initial positive blood culture showing gram-positive cocci, suspected catheter siterelated infection, skin or soft tissue infection, or hemodynamic instability. The source of fever was determined through review of electronic medical records, particularly infectious disease consult records. Patients were discharged in an afebrile state with neutrophil counts $\geq 1000 / \mathrm{mm}^{3}$.

\section{Outcome measures}

The primary outcome measure was to assess mortality during prophylaxis (at engraftment) and at 100 days after transplant. The secondary outcome measures included febrile episodes during pre-engraftment period, types and incidence of blood stream infection, and frequency of systemic antibiotic use. Additional secondary endpoints included time to engraftment, incidence of $C$. difficile infection, rate of ICU admission, length of stay in the ICU, length of transplant admission, and readmission rate in the first 100 post-transplant days. Outcomes were compared between patients who did and did not receive fluoroquinolone prophylaxis.

\section{Statistical analysis}

Patient characteristics were summarized using count and percentage for categorical variables and median with range for continuous variables. The statistical association between baseline characteristics and prophylaxis status was assessed. For continuous variables, the association was tested with the Kruskal-Wallis test, and either chi-squared or Fisher's exact tests were used for categorical variables. The Kaplan-Meier method was used to describe the survival without neutropenic fever where neutropenic fever was the endpoint; patients were censored at the time of their last contact, end of the study period, or death without neutropenic fever. Log-rank test was used to compare patient subgroups. Univariate and multivariate Cox proportional hazards regression models were fit to assess associations between patient characteristics and the neutropenic fever-free survival.

\section{Results}

\section{Baseline characteristics}

Between June 2013 and January 2015, 291 patients underwent AHSCT at the Karmanos Cancer Institute. Of these, 252 patients received norfloxacin prophylaxis and 39 patients did not. The baseline characteristics of both groups (without prophylaxis and with prophylaxis) 
are shown in Table 1. Both groups were comparable with regard to age at AHSCT, gender, race, and disease status at transplant.

The only significant differences between these two groups were diagnoses, prior treatment regimens, and conditioning regimens. Patients who received prophylaxis were more likely to have multiple myeloma (76\%) and received melphalan as preparative regimen $(p<0.001)$. In the group of patients who did not receive prophylaxis, more had NHL (46\%) and received rituximab and BEAM (R-BEAM) as preparative regimen $(p<0.001)$. The patients without prophylaxis group received higher number of prior treatment regimens compared to patients with prophylaxis [median $2(1-5)$ vs. $1(1-6) ; p=0.001$ ].

\section{Mortality}

There was no difference in the mortality during prophylaxis and at 100 days post-transplant in both groups $(p>0.99)$. None of the patients from either group died during prophylaxis period (prior to engraftment). Four patients from the prophylaxis group died as compared to none from without prophylaxis group within the first 100 post-transplant days. The causes of death were respiratory failure and septic shock. Another patient had sudden death of unknown etiology.

\section{ICU admission}

Twenty-eight (10\%) patients required ICU admission. Of these, 5 (13\%) patients without prophylaxis required ICU admission compared to $23(9 \%)$ patients with prophylaxis ( $p=$ $0.56)$. Majority of patients were transferred to ICU at a median of day +9 post-transplant. Patients without prophylaxis had ICU length of stay of median 4 days as compared to 7 days for patients with prophylaxis $(p=0.76)$. Seventeen patients were transferred to ICU due to infection. Two (40\%) patients without prophylaxis developed septic shock as compared to $10(43 \%)$ patients with prophylaxis $(p=0.23)$ (Table 2). Among non-infectious causes leading to ICU admission, atrial fibrillation (35\%) was the most common, followed by acute renal failure (24\%) and congestive heart failure (CHF) (14\%).

Median number of days of hospitalization after AHSCT was 14 days in both groups following AHSCT $(p=0.60)$. Three (8\%) patients without prophylaxis as compared to 29 (12\%) patients with prophylaxis were readmitted within the first 100 post-transplant days ( $p$ $=0.59)($ Table 2$)$.

\section{Neutropenic fever and blood stream infection}

The cumulative incidence of neutropenic fever among patients who underwent AHSCT was $79 \%$ (Table 3). The patients without fluoroquinolone prophylaxis (97\%) were more likely to develop neutropenic fever compared to patients with prophylaxis (77\%) $(p<0.005)$. Thirtyeight $(97 \%)$ patients without prophylaxis required systemic antibiotics as compared to 193 $(77 \%)$ patients with prophylaxis $(p=0.005)$. Similarly, group without prophylaxis utilized systemic antibiotics for median 9 days as compared to 7 days in patients with prophylaxis ( $p$ $=0.04)$. The group without prophylaxis used median 2 antibiotics as compared to 1 in patients with prophylaxis $(p=0.002)$. 
Clinically documented infection was noticed in two (5\%) patients without prophylaxis as compared to two $(1 \%)$ patients with prophylaxis $(p=0.13)$. Microbiologically documented infection occurred in $16(42 \%)$ patients without prophylaxis as compared to $67(35 \%)$ patients with prophylaxis $(p=0.50)$. Twenty $(53 \%)$ patients without prophylaxis and 124 (64\%) patients with prophylaxis had fever of unknown origin (FUO) $(p=0.24)$. Vascular catheter-related infection was the most common source of fever followed by skin/soft tissue infection. However, 40 patients with neutropenic fever had positive blood culture without any obvious source of fever identified. Hence, they were classified as "unknown" source of fever.

Seventy-seven (26\%) patients developed BSI. Fifteen (38\%) patients without prophylaxis developed BSI as compared to $62(25 \%)$ patients with prophylaxis $(p=0.10)$ (Table 3). Twelve (80\%) patients without prophylaxis developed BSI secondary to one organism as compared to 49 (79\%) patients with prophylaxis. Forty (14\%) patients developed single gram-positive bacteremia, $22(8 \%)$ patients developed single gram-negative bacteremia, and $15(5 \%)$ patients had polymicrobial infection (Table 4). The patients with prophylaxis demonstrated significantly higher gram-positive bacteremia (15\%) as compared to those without prophylaxis (8\%) $(p=0.002)$. Methicillin-sensitive Staphylococcus aureus (MSSA) and methicillin-resistant $S$. aureus (MRSA) blood stream infections were noticed in five and four patients with prophylaxis, respectively. Viridans streptococcus was the most common organism isolated in patients who received prophylaxis.

Five patients (13\%) without prophylaxis had $C$. difficile infections as compared to 19 (8\%) patients who received prophylaxis $(p=0.34)$. The patients without prophylaxis developed $C$. difficile infection at a median of day +2 (range, $1-7$ ) post-transplant, whereas the patients with prophylaxis had $C$. difficile infection at a median of day +5 (range, 1-48) posttransplant. The quinolone susceptibility for gram-negative bacteria showed resistance in one patient $(7 \%)$ from the without prophylaxis group and two patients (3\%) from the prophylaxis group.

\section{Kaplan-Meier and multivariate analysis}

Kaplan-Meier analysis of survival free from fever showed that patients without prophylaxis developed fever sooner ( 7 days, $95 \%$ CI 5-8 days) as compared to patients with prophylaxis ( 8 days, 95\% CI 8 days) $(p<0.001)$ (Fig. 1). In multivariable analysis, only the group who did not receive prophylaxis and type of conditioning regimen was independently associated with neutropenic fever (all $p<0.03$ ). The patients with $\mathrm{R} \pm \mathrm{BEAM}$ have a probability of developing neutropenic fever 2.2 times greater than those with melphalan (HR, 2.20; 95\% CI, 1.14 to $4.24 ; p=0.019$ ).

\section{Discussion}

Our study with norfloxacin prophylaxis demonstrates no difference in mortality during prophylaxis and at 100 days, frequency of ICU admission and stay, total length of hospitalization, and readmission rate between patients who did and did not receive prophylaxis. Although the role of fluoroquinolone prophylaxis in AHSCT recipients was previously explored in a few studies, morbidity data, particularly ICU admissions and length 
of stay, and readmission rate were not analyzed. Papadimitriou et al. showed that prophylaxis with oral ciprofloxacin and IV vancomycin in AHSCT patients reduced the incidence of neutropenic fever, and bacteremia, but no differences in hospitalization and allcause mortality were noted [16]. Similar results in terms of neutropenic fever and bacteremia were reported in other studies [11, 18, 19, 22]; nevertheless, mortality and morbidity were not evaluated. We believe that in the current era of improved cognizance of sepsis, timely use of antibiotics and better supportive care may have led to improved outcomes of patients with neutropenic fever. Moreover, non-infectious etiologies were equally accountable for ICU admission as noted in our study, and the clinical course of these patients may have been unaffected with fluoroquinolone prophylaxis. Length of hospitalization relies on full hematopoietic recovery, and G-CSF as well as stem cell dose plays an important role in accelerating engraftment [23]. Piñana et al. showed the length of neutropenia to be a significant risk factor for bacteremia in AHSCT [24], and we speculate that relatively rapid engraftment with the use of G-CSF could have shortened the duration of febrile episodes, infectious complications, morbidity, and eventually length of stay following transplant, making fluoroquinolone prophylaxis less important. Furthermore, all patients underwent inpatient transplant and were discharged after full hematopoietic recovery, which is heavily dependent on CD34+ cell dose, and thus, this explains the lack of difference in length of hospitalization between the two groups despite longer fever-free survival in patients with prophylaxis group.

Significantly higher frequency of gram-positive bacteremia was observed in patients with prophylaxis as compared without prophylaxis group $(p=0.002)$. Our study results are in line with the study by Bucaneve et al. [9] which showed more frequent gram-positive bacteremia in patients with levofloxacin prophylaxis. Of interest, the patients with prophylaxis had a higher rate of streptococcal bacteremia. Studies have documented gram-positive bacteremia especially viridans streptococci to be one of the risk factors for post-transplant morbidity and mortality. In post-transplant recipients, viridans streptococci may manifest as bacteremia/septicemia, endocarditis, and meningitis. Norfloxacin has inferior activity against gram-positive organisms [25], thus allowing colonization and septicemia with viridans streptococci. Similar results were observed in a prospective, double-blind, placebocontrolled study in leukemia patients where norfloxacin reduced gram-negative infection; however, no reduction was noted in frequency of gram-positive infection [26, 27]. In addition, fluoroquinolone itself is considered a risk factor for Streptococcus viridans infection [28]. Widespread use of fluoroquinolone has been associated with emergence of resistant gram-negative infection [13], and risk of emergence of resistant bacteria can exist even with short-term antibiotic prophylaxis [29].

Similar to prior studies, a substantial reduction in neutropenic fever in the patients who received norfloxacin prophylaxis was seen [12, 19]. Also, prior studies demonstrated lower rate of clinically or microbiologically documented infection [18] and higher rate of FUO with fluoroquinolone prophylaxis [16]. The failure to demonstrate statistical difference in other subcategories of fever in our study could probably be explained by the small sample size of the without prophylaxis group. In addition, in our study, the duration of systemic antibiotics for febrile episodes was shorter and the amount of antibiotics for neutropenic fever was lower in patients receiving prophylaxis. We hypothesize that the prophylactic 
antibiotic reduced the incidence of neutropenic fever and thus the amount and duration of antibiotic use for febrile episodes. Considering these results, the cost difference between prophylactic antibiotic use and total amount of antibiotics administered for neutropenic fever would be of interest. Notably, in a study by Papadimitriou et al., significantly higher cost was noted with prophylactic antibiotic as compared to empiric IV antibiotic therapy $(p<$ $0.0001)$ [16].

In our study, patients with $\mathrm{R} \pm \mathrm{BEAM}$ regimen have a probability of developing neutropenic fever 2.2 times greater than those with melphalan. In a study done by Veeraputhiran et al., BEAM was associated with increased fever, infections, and pneumonitis [30]. We think that BEAM induces severe mucositis, which is one of the risk factors for both local and systemic infections by providing portal of entry for the organisms [31].

Our study had two major limitations. It is a retrospective study limited to a single cancer center. Secondly, there is a possible selection bias because the decision to administer norfloxacin prophylaxis was based on individual physician discretion, and so, the two groups were not randomized. Thus, the group without prophylaxis was small. The economic impact of lower rate of neutropenic fever in the prophylaxis group remains to be determined.

In summary, norfloxacin prophylaxis was associated with significant decrease in antibiotic use post-transplant without significant impact on mortality during prophylaxis and at 100 days. Significant morbidities including ICU admission, ICU stay, and septic shock remained similar in both groups. Nevertheless, neutropenic fever is a morbid condition. Although frequency of neutropenic fever and antibiotic use were reduced with prophylaxis, norfloxacin use carries an increased risk of gram-positive bacteremia. It must be emphasized that the group without prophylaxis did not fare poorly in comparison to the group that received prophylaxis. Extrapolating from these data, we believe that prophylaxis with other available fluoroquinolones is likely to have similar overall outcome in the AHSCT population.

\section{Reference}

1. Petersen F, Thornquist M, Buckner C et al. (1988) The effects of infection prevention regimens on early infectious complications in marrow transplant patients: a four arm randomized study. Infection 16(4):199-208 [PubMed: 3053457]

2. Avril M, Hartmann O, Valteau-Couanet D, Brugieres L, Kalifa C, Lemerle J (1994) Antiinfective prophylaxis with ceftazidime and teicoplanin in children undergoing high-dose chemotherapy and bone marrow transplantation. Pediatr Hematol Oncol 11(1):63-73 [PubMed: 8155502]

3. Engels EA, Ellis CA, Supran SE et al. (1999) Early infection in bone marrow transplantation: quantitative study of clinical factors that affect risk. Clin Infect Dis 28(2):256-266 [PubMed: 10064241]

4. Hong J, Moon SM, Ahn HK et al. (2013) Comparison of characteristics of bacterial bloodstream infection between adult patients with allogeneic and autologous hematopoietic stem cell transplantation. Biol Blood Marrow Transplant 19(6):994-999 [PubMed: 23571460]

5. Kimura S, Akahoshi Y, Nakano H et al. (2014) Antibiotic prophylaxis in hematopoietic stem cell transplantation. A meta-analysis of randomized controlled trials. The Journal of infection 69(1):1325 [PubMed: 24583063]

6. Verhoef J (1993) Prevention of infections in the neutropenic patient. Clin Infect Dis 17(Suppl 2):S359-S367 [PubMed: 8274601] 
7. Patrick CC (1997) Use of fluoroquinolones as prophylactic agents in patients with neutropenia. Pediatr Infect Dis J 16(1):135-139 discussion 160-132 [PubMed: 9002124]

8. Del Favero A, Menichetti F (1993) The new fluorinated quinolones for antimicrobial prophylaxis in neutropenic cancer patients. Eur J Cancer 29A(Suppl 1):S2-S6

9. Bucaneve G, Micozzi A, Menichetti F et al. (2005) Levofloxacin to prevent bacterial infection in patients with cancer and neutropenia. N Engl J Med 353(10):977-987 [PubMed: 16148283]

10. Cullen M, Steven N, Billingham L et al. (2005) Antibacterial prophylaxis after chemotherapy for solid tumors and lymphomas. N Engl J Med 353(10):988-998 [PubMed: 16148284]

11. Sohn BS, Yoon DH, Kim S et al. (2012) The role of prophylactic antimicrobials during autologous stem cell transplantation: a single-center experience. Eur J Clin Microbiol Infect Dis 31(7):16531661 [PubMed: 22138805]

12. Garnica M, Nouer SA, Pellegrino FL, Moreira BM, Maiolino A, Nucci M (2013) Ciprofloxacin prophylaxis in high risk neutropenic patients: effects on outcomes, antimicrobial therapy and resistance. BMC Infect Dis 13:356 [PubMed: 23899356]

13. Cometta A, Calandra T, Bille J, Glauser MP (1994) Escherichia coli resistant to fluoroquinolones in patients with cancer and neutropenia. N Engl J Med 330(17):1240-1241 [PubMed: 8139646]

14. Pepin J, Saheb N, Coulombe MA et al. (2005) Emergence of fluoroquinolones as the predominant risk factor for Clostridium difficile-associated diarrhea: a cohort study during an epidemic in Quebec. Clin Infect Dis 41(9):1254-1260 [PubMed: 16206099]

15. Bartlett JG, Perl TM (2005) The new Clostridium difficile-what does it mean? N Engl J Med 353(23):2503-2505 [PubMed: 16322604]

16. Eleutherakis-Papaiakovou E, Kostis E, Migkou M et al. (2010) Prophylactic antibiotics for the prevention of neutropenic fever in patients undergoing autologous stem-cell transplantation: results of a single institution, randomized phase 2 trial. Am J Hematol 85(11): 863-867 [PubMed: 20882526]

17. Gafter-Gvili A, Fraser A, Paul M et al. (2012) Antibiotic prophylaxis for bacterial infections in afebrile neutropenic patients following chemotherapy. The Cochrane database of systematic reviews 1: CD004386 [PubMed: 22258955]

18. Wolska A, Robak T, Szmigielska-Kaplon A, Pluta A, Kopka P, Wierzbowska A (2012) Ciprofloxacin prophylaxis for patients undergoing high-dose chemotherapy and autologous stem cell transplantation (ASCT) —a single-center experience. Adv Med Sci 57(1):118-123 [PubMed: 22366265]

19. Vehreschild JJ, Moritz G, Vehreschild MJ et al. (2012) Efficacy and safety of moxifloxacin as antibacterial prophylaxis for patients receiving autologous haematopoietic stem cell transplantation: a randomised trial. Int J Antimicrob Agents 39(2):130-134 [PubMed: 22169408]

20. Freifeld AG, Bow EJ, Sepkowitz KA et al. (2011) Clinical practice guideline for the use of antimicrobial agents in neutropenic patients with cancer: 2010 update by the infectious diseases society of america. Clin Infect Dis 52(4):e56-e93 [PubMed: 21258094]

21. Hughes WT, Armstrong D, Bodey GP et al. (1997) 1997 guidelines for the use of antimicrobial agents in neutropenic patients with unexplained fever. Clin Infect Dis 25(3):551-573 [PubMed: 9314442]

22. Yeh SP, Chiu CF, Lo WJ et al. (2003) Low infectious morbidity in patients with heavily pretreated hematological malignancies receiving autologous peripheral blood stem cell transplantation without antimicrobial prophylaxis. Ann Hematol 82(1):24-29 [PubMed: 12574960]

23. Salazar R, Sola C, Maroto P et al. (1999) Infectious complications in 126 patients treated with high-dose chemotherapy and autologous peripheral blood stem cell transplantation. Bone Marrow Transplant 23(1):27-33 [PubMed: 10037047]

24. Pinana JL, Montesinos P, Martino R et al. (2014) Incidence, risk factors, and outcome of bacteremia following autologous hematopoietic stem cell transplantation in 720 adult patients. Ann Hematol 93(2):299-307 [PubMed: 23995612]

25. Razonable RR, Litzow MR, Khaliq Y, Piper KE, Rouse MS, Patel R (2002) Bacteremia due to viridans group Streptococci with diminished susceptibility to levofloxacin among neutropenic patients receiving levofloxacin prophylaxis. Clin Infect Dis 34(11):1469-1474 [PubMed: 12015693] 
26. Karp JE, Merz WG, Hendricksen C et al. (1987) Oral norfloxacin for prevention of gram-negative bacterial infections in patients with acute leukemia and granulocytopenia. A randomized, doubleblind, placebo-controlled trial. Ann Intern Med 106(1):1-7 [PubMed: 3538962]

27. Bow EJ, Rayner E, Louie TJ (1988) Comparison of norfloxacin with cotrimoxazole for infection prophylaxis in acute leukemia. The trade-off for reduced gram-negative sepsis. Am J Med 84(5): 847-854 [PubMed: 3284340]

28. Tunkel AR, Sepkowitz KA (2002) Infections caused by viridans streptococci in patients with neutropenia. Clin Infect Dis 34(11): 1524-1529 [PubMed: 12015700]

29. Gafter-Gvili A, Paul M, Fraser A, Leibovici L (2007) Effect of quinolone prophylaxis in afebrile neutropenic patients on microbial resistance: systematic review and meta-analysis. J Antimicrob Chemother 59(1):5-22 [PubMed: 17077101]

30. Veeraputhiran M, Jain T, Deol A et al. (2015) BEAM conditioning regimen has higher toxicity compared with high-dose melphalan for salvage autologous hematopoietic stem cell transplantation in multiple myeloma. Clin Lymphoma, Myeloma Leuk 15(9):531-535 [PubMed: 26166312]

31. McCann S, Schwenkglenks M, Bacon P et al. (2009) The Prospective Oral Mucositis Audit: relationship of severe oral mucositis with clinical and medical resource use outcomes in patients receiving high-dose melphalan or BEAM-conditioning chemotherapy and autologous SCT. Bone Marrow Transplant 43(2):141-147 [PubMed: 18776926] 


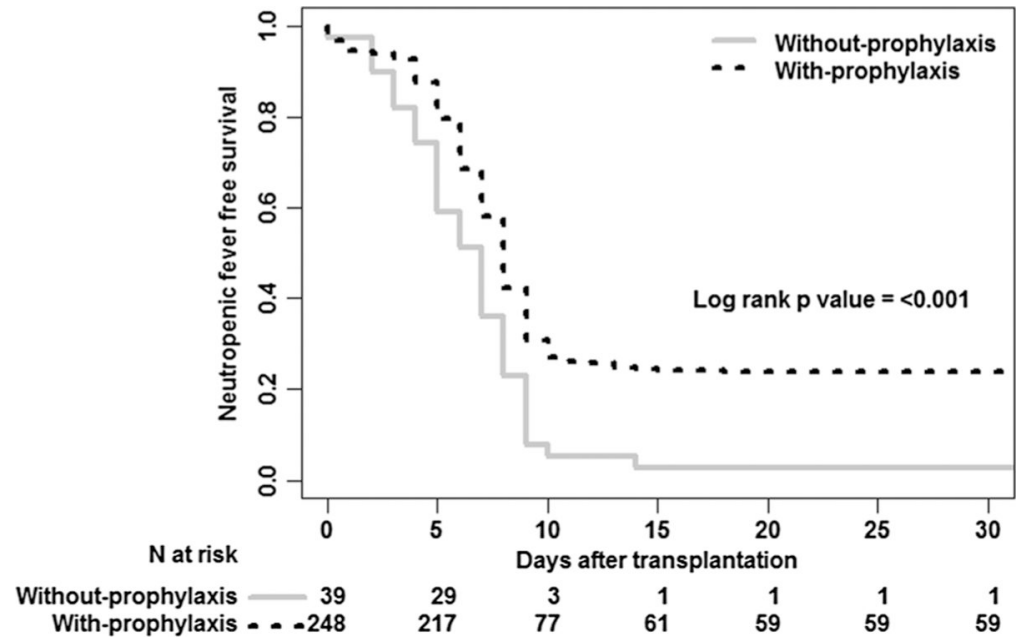

Fig. 1.

The neutropenic fever-free survival after autologous hematopoietic stem cell transplant comparing without prophylaxis vs. with prophylaxis group 


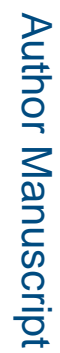

을

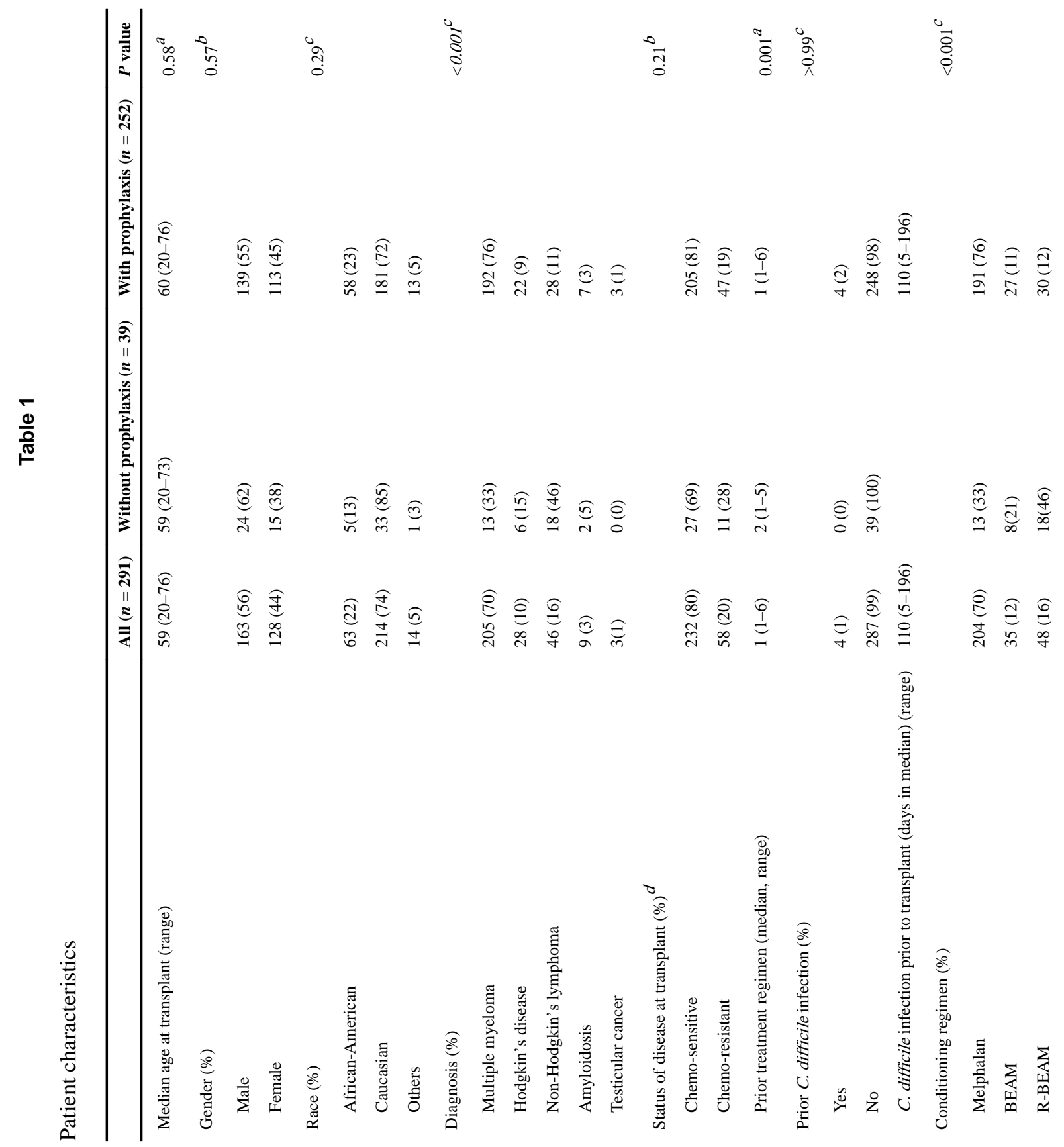

Support Care Cancer. Author manuscript; available in PMC 2019 December 02. 

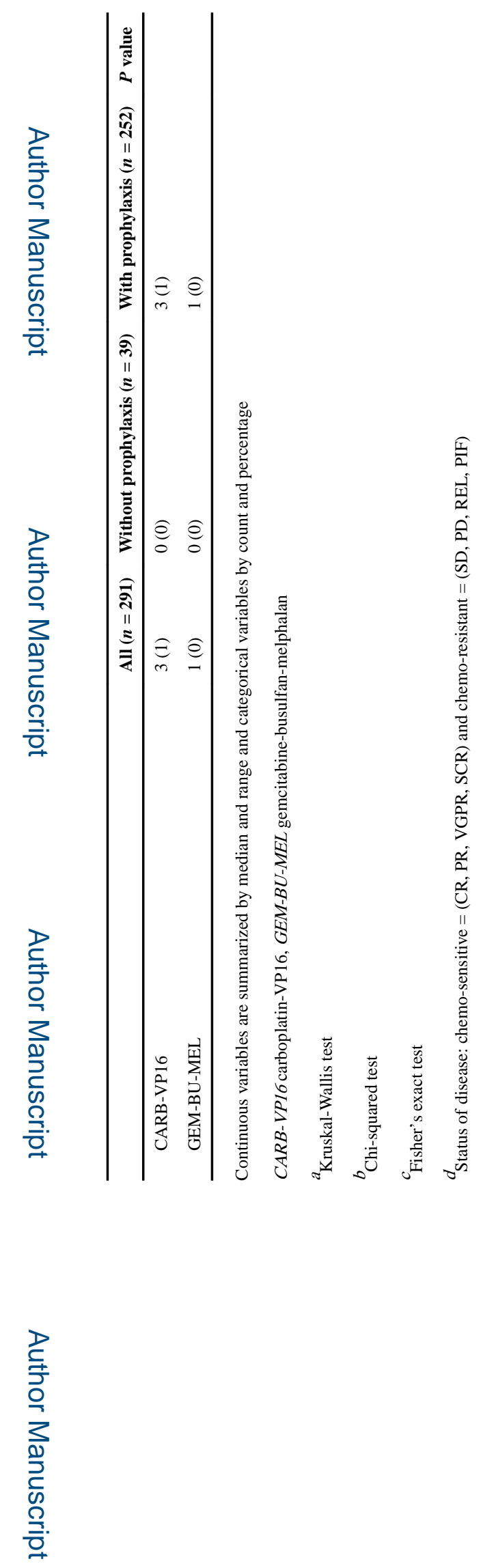


\section{롤 \\ 골}

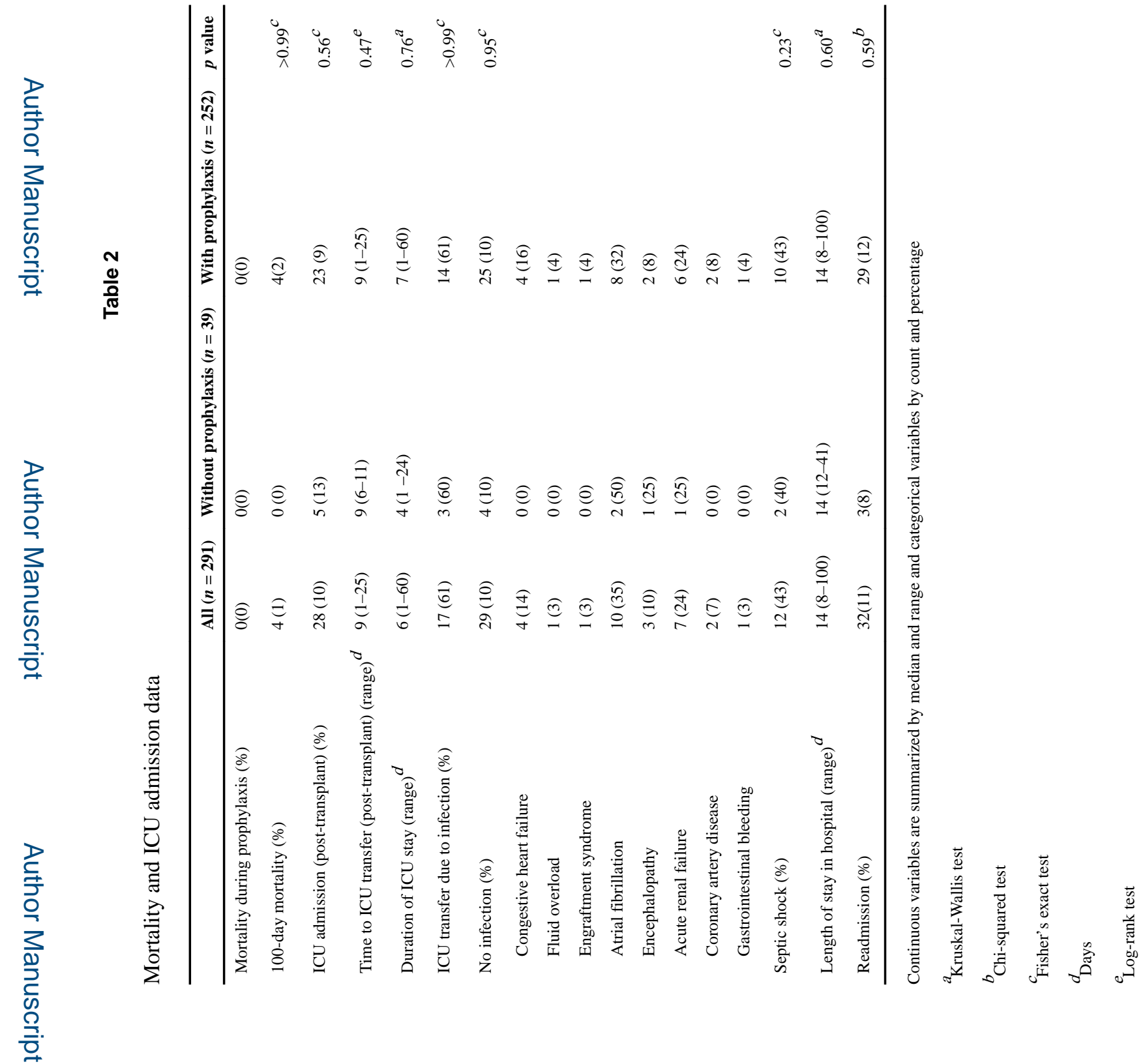

Support Care Cancer. Author manuscript; available in PMC 2019 December 02. 


\section{롬}

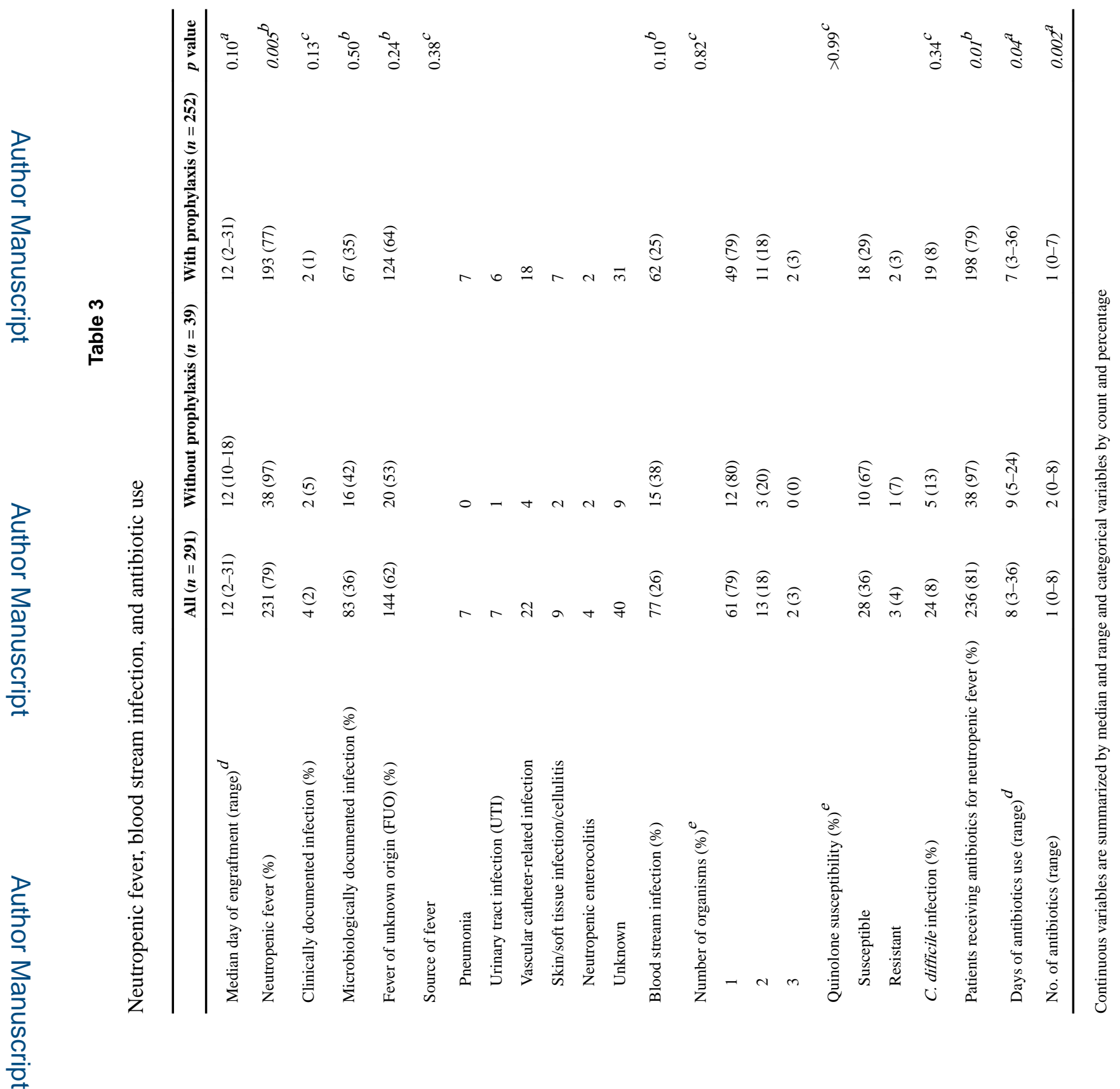




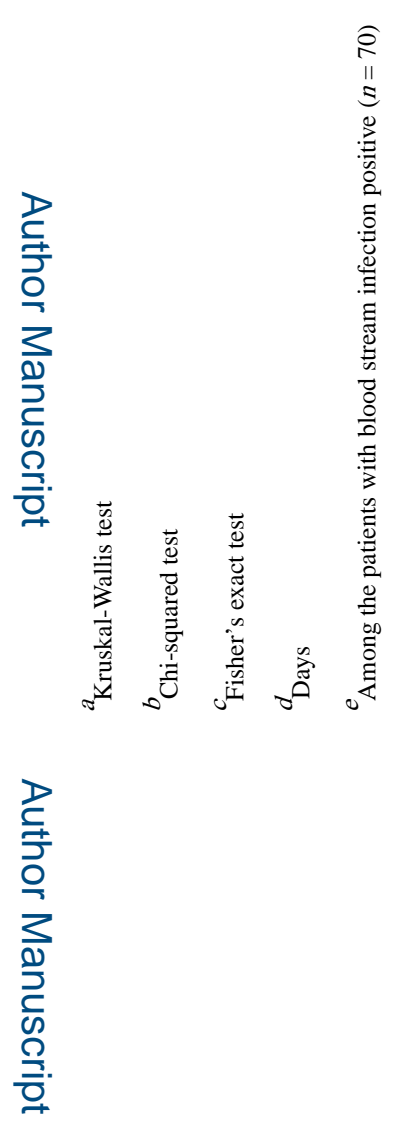

로을

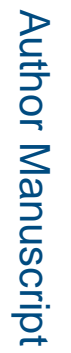

Support Care Cancer. Author manuscript; available in PMC 2019 December 02. 


\section{을 \\ 골}

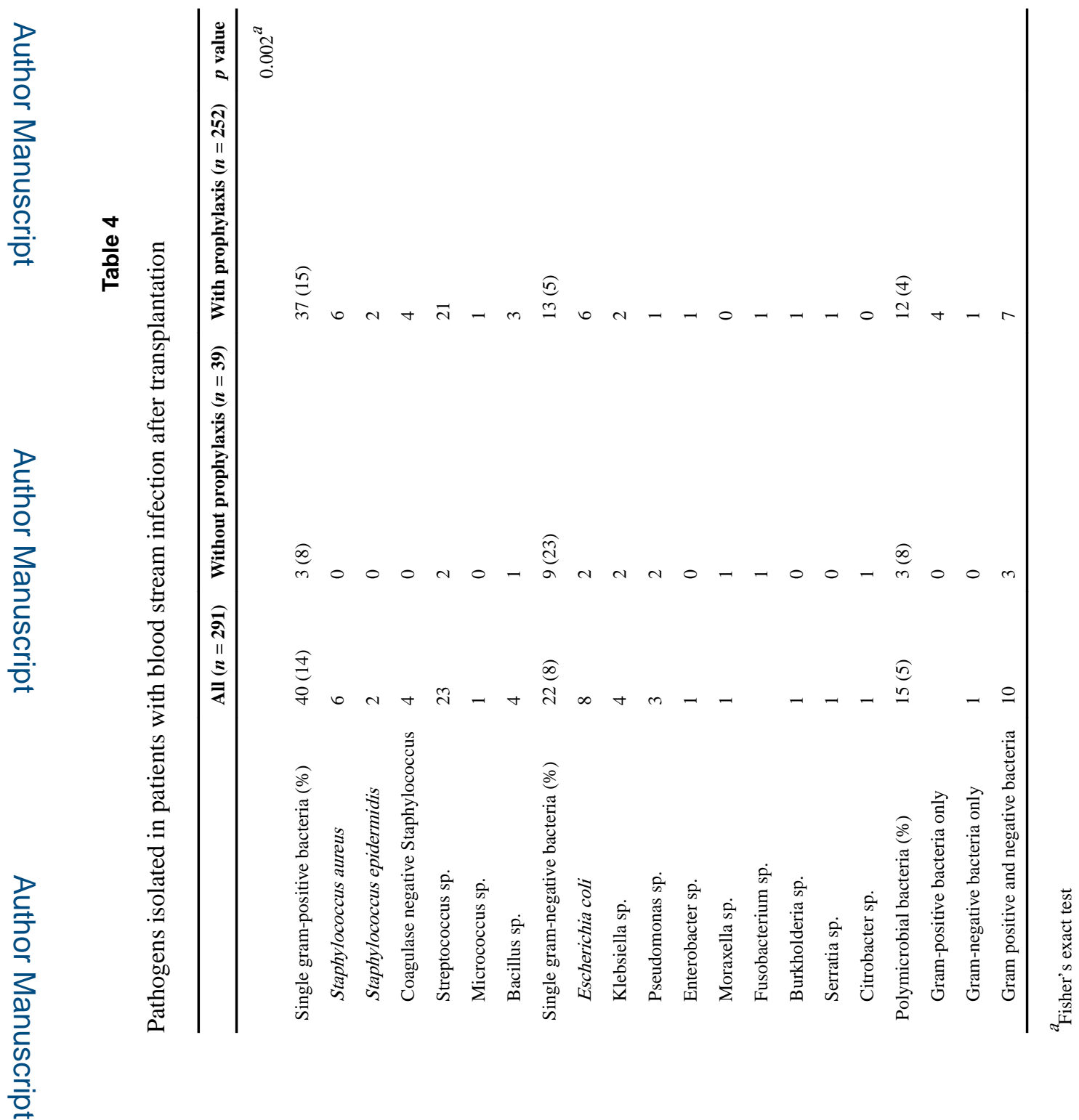

Support Care Cancer. Author manuscript; available in PMC 2019 December 02. 